\title{
Diabetes, its duration, and the long-term risk of abdominal aortic aneurysm: The Atherosclerosis Risk in Communities (ARIC) Study
}

\author{
Xuejuan Ning ${ }^{1}$, Ning Ding ${ }^{1}$, Shoshana H. Ballew ${ }^{1}$, Caitlin Hicks ${ }^{2}$, Josef Coresh ${ }^{1}$, Elizabeth \\ Selvin ${ }^{1}$, James Pankow ${ }^{3}$, Weihong Tang ${ }^{3}$, Kunihiro Matsushita ${ }^{1}$ \\ ${ }^{1}$ Johns Hopkins Bloomberg School of Public Health, Baltimore, MD, USA \\ 2Department of Surgery, Johns Hopkins University School of Medicine, MD, USA \\ ${ }^{3}$ University of Minnesota School of Public Health, Division of Epidemiology and Community \\ Health, Minneapolis, MN, USA
}

\begin{abstract}
Aims: We aimed at comprehensively evaluate the independent association of diabetes and its duration with incident abdominal aortic aneurysm (AAA) and aortic diameter.
\end{abstract}

\begin{abstract}
Methods and Results: We prospectively studied incident AAA according to baseline glycemic status (diabetes, prediabetes, normal glycemia) in 13,116 ARIC participants (1990-1992) and the time-varying exposure of duration post incident diabetes in 11,675 participants (1987-1989) using Cox models. Additionally, we cross-sectionally explored ultrasound-based abdominal aortic diameter by glycemic status and cumulative duration of diabetes in 4710 participants (2011-2013) using linear regression models. Over $\sim 20$ years of follow-up, diabetes ( $v S$. normal glycemia) at baseline was independently associated with lower AAA risk (489 cases) (hazard ratio: 0.71 [95\%CI $0.51-0.99]$ ), especially after 10 years (hazard ratio: 0.58 [0.38 - 0.87]). Prediabetes did not demonstrate an independent association. The inverse association was more evident with longer duration of diabetes ( $p$ for trend $=0.045$ ), with 30-50\% lower risk in eight years after diabetes diagnosis. The cross-sectional analysis demonstrated smaller aortic diameters with longer duration of diabetes (e.g., $-0.76 \mathrm{~mm}[-1.24,-0.28]$ in diabetes with $8-12$ years) compared to non-diabetes, whereas prediabetes consistently showed nominally greater diameter.
\end{abstract}

\footnotetext{
Corresponding author: Kunihiro Matsushita, Department of Epidemiology, Johns Hopkins Bloomberg School of Public Health and Welch Center for Prevention, Epidemiology, and Clinical Research, 2024 E. Monument St., Suite 2-600, Baltimore, MD, 21287. kuni.matsushita@jhu.edu.

Author contributions

Dr. Matsushita took responsibility for the integrity of the data and the accuracy of the data analysis. K.M. and X.N. developed the study concept and study design. X.N., K.M. conducted data analysis and interpretation. X.N., K.M. drafted the manuscript. All authors provided critical feedback and helped shape the research, analysis and manuscript.

Publisher's Disclaimer: This is a PDF file of an unedited manuscript that has been accepted for publication. As a service to our customers we are providing this early version of the manuscript. The manuscript will undergo copyediting, typesetting, and review of the resulting proof before it is published in its final form. Please note that during the production process errors may be discovered which could affect the content, and all legal disclaimers that apply to the journal pertain.

Conflict of interest

The authors declared they do not have anything to disclose regarding conflict of interest with respect to this manuscript.

All authors declare no other conflict of interests in any form.
} 
Conclusions: Diabetes, especially with longer duration, but not prediabetes, was independently associated with lower risk of AAA and smaller aortic diameter. Our findings suggest that long lasting clinical hyperglycemia plays an important role in the reduced AAA risk, and the reduced aortic diameter may be a structural mechanism behind this paradoxical association.

\section{Graphical Abstract}
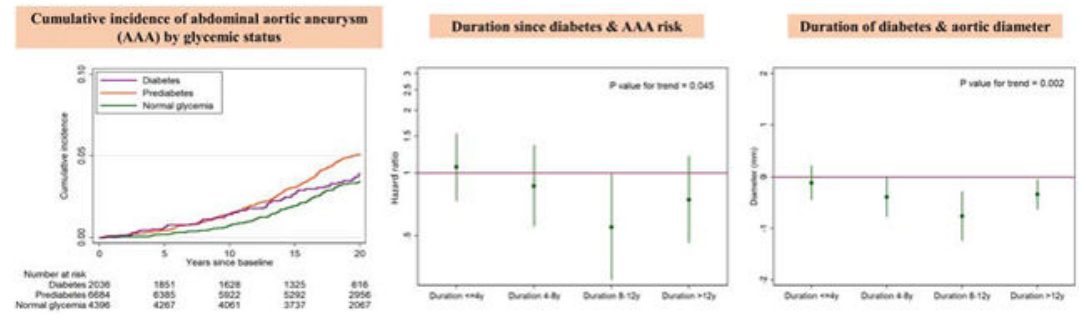

\section{Keywords}

diabetes; prediabetes; abdominal aortic aneurysm; aortic diameter

\section{Introduction}

Abdominal aortic aneurysm (AAA) affects $\sim 5 \%$ of men and $\sim 1 \%$ of women in the U.S. ${ }^{1}$ Since the fatality rate is $75 \%-90 \%$ once it ruptures, ${ }^{1}$ it is critical to modify risk factors and prevent the development and progression of AAA. Major cardiovascular risk factors, such as older age, male sex, cigarette smoking, and hypertension, are known to increase the risk of AAA. $^{1-3}$

It is intriguing that diabetes mellitus, one of the strongest cardiovascular risk factors, ${ }^{4}$ has been associated with lower risk of AAA. ${ }^{5,6}$ Since the first report of this paradoxical association in $1997,{ }^{7}$ a number of subsequent studies have consistently reported the inverse association between diabetes and AAA risk..$^{2,4,8-11}$ Moreover, slower aneurysmal growth rate has been reported in diabetes compared to non-diabetes. ${ }^{12}$

However, the independent association of the full spectrum of glycemic status including prediabetes with AAA risk has not been characterized. Also, in the vast majority of previous studies, the definition of diabetes relied on self-reported diagnosis or medical records, which could misclassify the status of diabetes. ${ }^{13}$ Moreover, whether a dose-response relationship exists between the duration of diabetes and AAA risk has not been extensively investigated.

In this community-based cohort study, the Atherosclerosis Risk in Communities (ARIC) Study, we sought to comprehensively assess the independent association of glycemic status and AAA risk. Specifically, we evaluated glycemic status (normal glycemia, prediabetes and diabetes) using fasting glucose and hemoglobin $\mathrm{A} 1 \mathrm{c}(\mathrm{HbA} 1 \mathrm{c})$ in addition to a clinical history of diabetes. ${ }^{14}$ We also examined the duration of diabetes as a time-varying exposure. Moreover, we supplemented these analyses with a cross-sectional analysis of ultrasoundbased abdominal aortic diameter. 


\section{Materials and methods}

\section{Study population}

The ARIC Study enrolled 15,792 participants aged 45-64 years from four U.S. communities during 1987-1989 (visit 1). ${ }^{15}$ All participants provided informed consent. Then, three shortterm follow-up visits (visits 2-4) were held approximately every three years. Visit 5 was conducted in 2011-2013. The current study consisted of a prospective analysis with incident AAA as the outcome and a cross-sectional analysis with aortic diameter as the outcome variable.

The prospective analysis explored glycemic status as a time-fixed exposure and the duration since incident diabetes as a time-varying exposure. For the former, visit 2 (1990-1992) served as the baseline, because $\mathrm{HbA1c}$ was available in addition to fasting glucose. Of the 14,348 participants at visit 2 , we excluded races other than White or Black $(n=42)$; prevalent AAA $(n=16)$; non-fasting or missing fasting status $(n=437)$; missing variables $(n=737)$, leaving 13,116 participants. For the analysis with diabetes duration as a time-varying exposure, we used incident diabetes ascertained only after visit 1 to avoid relying on participants' recall regarding diabetes duration prior to visit 1 . Applying the aforementioned exclusion criteria and excluding prevalent diabetes at visit $1(n=1,867)$, the final study sample included 11,675 participants.

The cross-sectional analysis was based on visit 5 (2011-2013) when abdominal ultrasound was performed. Of the 6,538 participants at visit 5 , we excluded races other than White or Black ( $\mathrm{n}=18)$; missing or uninterprSupplementary Table aortic diameter measurements $(\mathrm{n}=438)$; non-fasting or missing fasting status $(\mathrm{n}=342)$; or missing variables $(\mathrm{n}=1,030)$, leaving 4,710 participants.

\section{Glycemic status and durations of diabetes}

Glycemic status was categorized as diabetes, prediabetes, and normal glycemia at visit 2 . Diabetes was defined based on self-reported physician diagnosis, or self-reported antidiabetic medication use, or fasting glucose $\geq 126 \mathrm{mg} / \mathrm{dL}$, or HbA1c $\geq 6.5 \% .{ }^{14}$ Prediabetes was defined as fasting glucose of $100-125 \mathrm{mg} / \mathrm{dL}$, or HbA1c of 5.7\%-6.4\% ${ }^{14}$ among those without diabetes. Normal glycemia referred to neither diabetes nor prediabetes.

Glucose was measured using hexokinase method using fresh serum samples. HbA1c was measured using Diabetes Control and Complications Trial assay (Tosoh 2.2 Plus HPLC instrument and Tosoh G7 HPLC instrument) using whole blood samples stored at $-70^{\circ} \mathrm{C} .{ }^{16}$

The duration of diabetes as a time-varying exposure was based on incident diabetes after visit 1 by applying the same criteria above using data at follow-up visits and data from annual follow-up interviews (semiannually since 2012) about self-reported physician diagnosis or glucose-lowering medication use. We categorized the duration of diabetes into $\leq 4$ years, $4-8$ years, $8-12$ years, $>12$ years because 4 -year interval was the shortest period allowing us to obtain reliable estimates given that the majority of participants did not have updated data within 3 years from visit 1 through visit 4 . 
For the cross-sectional analysis, glycemic status was defined using the same criteria noted above by accounting for information at visit 5 . However, participants who met criteria for diabetes at any prior visits were also categorized as diabetes. ${ }^{17} \mathrm{We}$ also explored the duration of diabetes, with the same duration categories as the prospective analysis.

\section{Covariates}

The same approach was taken to collect data at visits 1 and 2. Age, sex, race, educational level (advanced, intermediate, and basic), cigarette pack-years, and alcohol use were selfreported. Body mass index (BMI) was calculated as body weight (in kilograms) divided by height (in meters) squared. Blood pressure was measured three times after 5 min of rest using a random-zero sphygmomanometer, and the average of the last two readings was used in the analysis. Total and high-density lipoprotein (HDL) cholesterols were determined using standardized enzymatic assays. Estimated glomerular filtration rate (eGFR) was calculated using serum creatinine. ${ }^{18}$ Medication use over the prior two weeks was verified by medication containers. History of coronary heart disease was defined by a self-reported history or electrocardiogram indicative of myocardial infarction at visit 1 , or subsequent adjudicated cases. ${ }^{19}$ History of stroke was similarly defined as a self-reported history at visit 1 or adjudicated cases afterwards. History of peripheral artery disease was defined by a selfreported history at visit 1 and subsequent hospitalizations with its diagnosis during the follow-up. The same covariates were similarly collected at visit 5, with a few exceptions: medication use identified for the past four weeks and blood pressure measured with an oscillometric device (OMRON HEM-907XL). Cigarette pack-years was not recorded at visit 5 , and thus we used data at visit 4 .

\section{Incident AAA and aortic diameters}

The details of identifying incident clinical AAAs in ARIC have been published elsewhere. ${ }^{20,21}$ Briefly, through annual telephone interviews, any interim hospitalizations or deaths were identified, and medical records or death certificates were verified in case of such events. The ARIC Study also conducted active surveillance of local hospitals. Additionally, the ARIC Study linked participant identifiers to the Centers for Medicare and Medicaid Services (CMS) data to supplement additional inpatient and outpatient events for those aged $\checkmark 65$ years. Clinical AAA cases were based on the ICD-9 codes of 441.3 (AAA, ruptured), 441.4 (AAA, without rupture), 38.44 (AAA, resection with replacement) or 39.71 (AAA, endovascular repairment) or cause of death with the ICD-9 or 10 codes of 441.3, 441.4, I71.3 (AAA, ruptured) or I71.4 (AAA, without rupture) and were defined as either hospitalizations from any source or two outpatient claims at least a week apart.

Abdominal ultrasound was performed at visit 5 (2011-2013) using Philips iE33 high resolution duplex scanner and Philips $\mathrm{C} 5-1$ transducer. ${ }^{20,21}$ Certified technicians acquired images at the proximal aorta (just below the superior mesenteric artery), the mid aorta $(2 \mathrm{~cm}$ below the renal arteries), the distal aorta $(1 \mathrm{~cm}$ above the aortic bifurcation), and the place of maximal aortic diameter other than those three locations if any and measured anteriorposterior and transverse aortic diameter. We analyzed the larger diameter at each site. 


\section{Statistical analyses}

Baseline characteristics were compared by glycemic status at visit 2 and visit 5 using Chisquared test. For the prospective analysis, we first estimated the cumulative incidence of AAA by glycemic status at visit 2 using the Kaplan-Meier method. Then, we estimated the hazard ratios (HRs) of incident AAA using Cox regression models. Since we observed nonproportional hazards over time, we stratified by follow-up time < and $\geq 10$ years. We constructed two models to account for potential confounders. Model 1 was unadjusted, and Model 2 adjusted for age, sex, race, education, BMI, height, cigarette pack-years, alcohol use, systolic blood pressure, diastolic blood pressure, antihypertensive medication, total cholesterol, HDL cholesterol, cholesterol-lowering medication, eGFR, prevalent coronary heart disease, prevalent stroke, and prevalent peripheral artery disease. Since our interest was the etiological association of diabetes with AAA, we prioritized presenting Model 2 results. Several sensitivity analyses were conducted by treating mortality as a competing risk and stratifying according to the use of antidiabetic medications. To test the consistency of our primary results, we also explored $\mathrm{HbA1c}$ levels $(<5.7 \%, 5.7-6.4 \%, 6.5-6.9 \%, 27.0 \%)$ at visit 2 and repeated the main analysis among 10,459 eligible participants at visit 4 .

We subsequently examined the duration of diabetes ( $\leq 4$ years; $4-8$ years; 8-12 years; >12 years) as a time-varying exposure. We fitted Cox models and adjusted for the same covariates but in a time-varying fashion. Linear trend was tested by treating duration as a continuous variable.

For the cross-sectional analysis at visit 5, we first quantified the aortic diameter by glycemic status, and then by cumulative duration of diabetes. We ran multivariable linear regression models adjusting for the same set of covariates described above at visit 5. Unique to visit 5 data, we were able to specifically explore subgroups by selected anti-diabetic medications such as metformin and sulfonylureas. Other medications (insulin, thiazolidinediones, and dipeptidyl peptidase-4 inhibitors) were not explored due to small numbers of participants. Considering the potential selection bias of our visit 5 participants vs. those who could not attend visit 5 (although they were alive), we conducted an inverse probably weighting analysis for the probability of attending visit 5 conditioning on the covariates that were included in Model 2 as well as their glycemic status at visit 2. We also modeled the HbA1c categories at visits 2 and 5 .

All statistical analyses were conducted using Stata SE, version 14 (Stata Corp, College Station, TX), and a two-tailed $p$ value $<0.05$ was considered statistically significant.

\section{Results}

\section{Baseline glycemic status and incident AAA}

The mean age of the 13,116 visit 2 participants was 57.0 (SD 5.7), $55.7 \%$ were female, and $23.4 \%$ were of Black race. The prevalence of prediabetes and diabetes were $51.0 \%$ and $15.5 \%$, respectively. Compared to participants with normal glycemia, those with prediabetes and diabetes were more likely to be older, male, Black, taller, smoking more cigarettes, less educated, and more likely to have poorer risk profiles (e.g. higher mean BMI, blood 
pressures, and total cholesterol, lower mean HDL cholesterol, and higher prevalence of coronary heart disease, stroke and peripheral artery disease) (Table 1).

During a median follow-up of 19.7 years, there were 489 incident AAA cases. Although small in absolute differences, the cumulative incidence of AAA was highest in diabetes in the first $\sim 10$ years of follow-up (purple line in Figure 1). After $\sim 10$ years of follow-up, participants with prediabetes showed the highest cumulative incidence of AAA (orange line in Figure 1). The cumulative incidence became similar for participants with diabetes and normal glycemia around 20 years of follow-up. Generally similar patterns were seen when we treated mortality as a competing risk (Supplementary Fig. 1) and investigated visit 4 as baseline (Supplementary Fig. 2).

Over the entire follow-up, diabetes was not associated with AAA in a crude model but showed a significantly lower risk after adjusting for potential confounders included in Model 2 (HR: 0.71 [95\%CI 0.51-0.99] in Model 2) (Table 2). Stratifying by follow-up time, the inverse independent association was evident after 10 years of follow-up (HR: 0.58 [95\%CI $0.38-0.87$ ] in Model 2). On the other hand, prediabetes demonstrated significantly positive associations with AAA risk only in unadjusted models but not in multivariable models regardless of follow-up time. The results were largely consistent in race subgroups (Supplementary Table 1). When we explored the categories of HbA1c, HbA1c 5.7-6.4\% showed a positive association with incident AAA (only statistically significant in Model 1), whereas $\mathrm{HbA} 1 \mathrm{c} \geq 7.0 \%$ was associated with a lower risk of AAA in Model 2 (Supplementary Table 2).

The results were similar after accounting for mortality as a competing risk (Supplementary Table 3) and when we subdivided diabetic participants by the use of antidiabetic medications (Supplementary Table 4). When we compared participants with diabetes duration $<3$ years (newly diagnosed diabetes at visit 2 ) or $\geq 3$ years (prevalent diabetes at visit 1), elevated risk of AAA within the first 10 years was similar in these two groups but the inverse associations with AAA after 10 years of follow-up was more evident in the group with duration $\geq 3$ years than $<3$ years (Supplementary Table 5).

\section{Duration of diabetes and incident AAA}

Among 11,675 participants without diabetes at visit 1, there were 443 incident AAA cases over a median follow-up of 22.7 years. We observed an inverse association of longer duration of diabetes with incident AAA after adjusting for potential confounders in Model 2 ( $\mathrm{p}$-for-trend $=0.045$ in Figure 2). Although duration of diabetes $>12$ years did not reach statistical significance, diabetes duration of $>8$ years had 30\%-50\% lower risk of AAA compared to non-diabetes. Largely similar patterns were observed in Model 1 (Supplementary Fig. 3) although the non-significant positive association of the diabetes duration $\leq 4$ years with incident AAA was more evident in Model 1 compared to Model 2.

\section{Glycemic status and aortic diameters}

At visit 5, 45.0\% of participants had prediabetes and 38.0\% had diabetes. Prediabetes and diabetes demonstrated worse risk factor profiles relative to the normal glycemia group (Supplementary Table 6). 
In crude models (Model 1), compared to normal glycemia, participants with prediabetes and diabetes showed consistently larger aortic diameters (Supplementary Table 7). The differences were more evident for prediabetes than diabetes at almost all sites. After adjusting for potential confounders (Model 2), prediabetes demonstrated larger diameters at the proximal aorta with borderline significance $(+0.23$ [95\%CI $-0.01-+0.48], \mathrm{p}=0.06)$, whereas diabetes had smaller diameter than normal glycemia at all sites, with the most evident difference at distal aorta $(-0.26$ [95\% CI $-0.56-+0.03], \mathrm{p}=0.08)$. The patterns were consistent after accounting for probability of attending visit 5 examination (Supplementary Table 8). When we investigated the categories of $\mathrm{HbA1c}$, the aortic diameters were generally smaller in participants with higher HbA1c levels at both visit 5 and visit 2 (Supplementary Table 9 and Supplementary Table 10).

Longer duration of diabetes was associated with smaller aortic diameters, which became more prominent after 8 years (Figure 3 and Supplementary Table 11). Diabetes duration 812 years had $0.76 \mathrm{~mm}(95 \% \mathrm{CI} 0.28$ - 1.24) smaller maximum diameter compared to nondiabetes (Figure 3). We found generally consistent results when stratified by race (Supplementary Table 12), the use of anti-diabetic medications (Supplementary Table 13) and different types of medications (i.e., metformin and sulfonylureas) (Supplementary Table s 14 and 15).

\section{Discussion}

This community-based cohort with over $\sim 20$ years of follow-up confirmed that diabetes was overall associated with lower AAA risk. However, this association was not simple. Baseline diabetes was not independently associated with incident AAA in the first 10 years of followup but was inversely associated with AAA incidence afterwards. The inverse association was more evident in diabetes with longer duration, with 30-50\% lower risk of AAA in diabetes with a duration $>8$ years compared to non-diabetes. The cross-sectional analysis with aortic diameter supported the prospective analysis. Longer duration of diabetes was independently associated with smaller diameter. In contrast, prediabetes did not demonstrate independent associations with AAA and aortic diameter. The results were consistent in sensitivity analyses with visit 4 as baseline, mortality as a competing outcome, weighting the attrition at visit 5 , and the focus on antidiabetic medications.

Although there have been a dozen prospective studies reporting an inverse association between diabetes and AAA, ${ }^{2-4,11,21-29}$ several unique aspects of our study should be noted. First, we observed distinct associations of diabetes with incident AAA in $<$ and $\geq 10$ years of follow-up. We are aware of only one study reporting a similar pattern. ${ }^{30}$ Second, to our knowledge, this is the first study evaluating duration of diabetes as a time-varying exposure for incident AAA. Third, we explored cumulative duration of diabetes and aortic diameter. Fourth, glycemic status was comprehensively defined by fasting glucose, HbA1c, and selfreported clinical history. Finally, our study uniquely explored incident AAA and abdominal aortic diameter in the same study population.

It is yet unclear why diabetes results in lower risk of AAA despite its well-known pathophysiological contribution to other cardiovascular diseases. Nonetheless, given that a 
lower risk of AAA was not seen in prediabetes and diabetes in earlier follow-up or with short duration in our study, long lasting clinical hyperglycemia (at diabetes range) seems to play a crucial role in this paradoxical relationship.

Several possible pathophysiological mechanisms linking hyperglycemia to the decreased risk of AAA have been proposed. For example, hyperglycemia is reported to increase synthesis and decrease degradation of extracellular matrix, resulting in thicker intima-media layer of aortic wall, ${ }^{31,32}$ which may prevent the dilation of AAA. In addition to hyperglycemia, advanced glycation end products, ${ }^{33}$ also are likely to reduce the degradation of extracellular matrix. ${ }^{34}$

Some experts suggest that elevated aortic stiffness due to hyperglycemia may prevent the development and growth of AAA. ${ }^{35,36}$ Also, it has been argued that high mortality risk in diabetic patients makes them less likely to develop AAA. However, these two theories seem unlikely to fully explain the inverse association between diabetes and AAA since diabetes is not the only condition that increases both aortic stiffness and mortality risk. For example, hypertension is another representative clinical condition but is known as a potent risk factor for AAA. Indeed, in our data, hypertension and pre-hypertension were positively associated with incident AAA in a graded manner (Supplementary Fig. 4). Also, the inverse association of diabetes and AAA risk was confirmed in survival models accounting for mortality as a competing outcome.

Metformin recently attracted attention as a potential explanation behind lower risk of AAA in diabetes. An animal study demonstrated that AAA enlargement was prevented with metformin. ${ }^{37} \mathrm{~A}$ few human studies have shown that diabetic patients with metformin prescriptions had lower incidence and slower growth rate of AAA compared to those without metformin prescription. ${ }^{38-40}$ However, it were challenging for these studies to disentangle the impact of diabetes itself (only diabetic patients were included), its duration, and antidiabetic medications on AAA. Indeed, lower risk and slower growth rate of AAA were actually seen with prescriptions of other antidiabetic medications (e.g., sulfonylurea and thiazolidinedione) as well in those studies. Moreover, the inverse association between diabetes and AAA was reported even before metformin became widely prescribed in the US. 6,41 Thus, the protective effect of metformin on AAA still remains debatable and ultimately should be investigated in clinical trials.

We observed that diabetes showed consistently smaller aortic diameters than prediabetes or normal glycemia at a given level of potential confounders. Smaller aortic diameter was particularly evident in diabetes with longer duration. A number of studies have reported "negative remodeling" (decreasing vascular area and diameter) of coronary arteries in diabetic patients, ${ }^{42-46}$ resulting in the typical manifestation of diffuse arterial narrowing in this clinical population. Although the exact pathophysiological mechanisms are yet unknown, interestingly, two studies demonstrated that diabetic patients had smaller aortic root diameters compared to those without diabetes. ${ }^{47,48}$

Although this concept of reduced aortic diameter needs to be confirmed in other studies, it can actually explain an unsolved mystery in vascular medicine about diverging effects of 
diabetes on increasing the risk of peripheral artery disease vs. decreasing the risk of AAA, despite the close relation between peripheral artery disease and AAA in general ${ }^{49} \mathrm{It}$ is intuitive that negative arterial remodeling due to long-lasting diabetes would contribute to the development of peripheral artery disease. On the other hand, negative aortic remodeling may happen to be protective against AAA.

Shedding light on "negative remodeling" as a potential structural mechanism behind lower AAA risk in diabetes has an important implication. Although a number of studies have been conducted to discover "protective" pathways of diabetes against AAA,,${ }^{50-52}$ our findings indicate that there may not be such a beneficial pathway and lower risk of AAA may be just a fortunate consequence of devastating negative remodeling in diabetic patients. Thus, we should prioritize the investigation of pathophysiological mechanisms behind negative vascular remodeling in diabetes.

Our findings in prediabetes deserve some discussion. Prediabetes demonstrated a higher risk of AAA than normal glycemia in unadjusted models, which were somewhat expected since the major risk factors of AAA (i.e., smoking and higher blood pressure) were more prevalent in prediabetes than normal glycemia. Indeed, once we accounted for these potential confounders, prediabetes was no longer associated with AAA risk (Model 2 in Table 2). In contrast to diabetes, prediabetes has not been extensively evaluated in the context of AAA, and thus further investigation would be needed.

Several limitations of our study should be noted. First, incident AAA cases were determined using ICD codes, which could underestimate the incidence. Second, because abdominal ultrasound was not performed at visit 1 or 2 , some of the incident AAA cases could have had undiagnosed AAA at baseline. However, such cases might be rare considering the low prevalence of AAA before age $65 .{ }^{1}$ Third, abdominal ultrasound is technician dependent, potentially resulting in some misclassification and reduced statistical power. ${ }^{53}$ Fourth, the date of incident diabetes was defined based on the date of first ascertainment (date of ARIC exam or interview), as participants were not asked to provide the date of diagnosis by a physician. Fifth, oral glucose tolerant test (OGTT) was not repeatedly performed in ARIC, therefore some participants with prediabetes were not captured, and we were unable to differentiate type 1 and type 2 diabetes. Finally, we could not rule out the possibility of residual confounding (e.g., family history of AAA).

In conclusion, diabetes, especially with longer duration, but not prediabetes, was independently associated with lower risk of AAA and smaller aortic diameter. Our findings suggest that long lasting clinical hyperglycemia plays an important role in the reduced AAA risk and the reduced aortic diameter may be a structural mechanism behind this paradoxical association.

\section{Supplementary Material}

Refer to Web version on PubMed Central for supplementary material. 


\section{Acknowledgements}

The Atherosclerosis Risk in Communities study has been funded in whole or in part with Federal funds from the National Heart, Lung, and Blood Institute, National Institutes of Health, Department of Health and Human Services, under Contract nos. (HHSN268201700001I, HHSN268201700002I, HHSN268201700003I, HHSN268201700005I, HHSN268201700004I). The present study was supported by NHLBI grant R01HL103695.

The authors thank the staff and participants of the ARIC study for their important contributions.

This work is supported by the NIH grant listed in the Acknowledgement section.

\section{Reference}

1. LeFevre ML, U. S. Preventive Services Task Force. Screening for abdominal aortic aneurysm: U.S. Preventive Services Task Force recommendation statement. Ann Intern Med 2014;161(4):281-290. [PubMed: 24957320]

2. Kent KC, Zwolak RM, Egorova NN, Riles TS, Manganaro A, Moskowitz AJ, Gelijns AC, Greco G. Analysis of risk factors for abdominal aortic aneurysm in a cohort of more than 3 million individuals. J Vasc Surg 2010;52(3):539-548. [PubMed: 20630687]

3. Iribarren C, Darbinian JA, Go AS, Fireman BH, Lee CD, Grey DP. Traditional and novel risk factors for clinically diagnosed abdominal aortic aneurysm: the Kaiser multiphasic health checkup cohort study. Ann Epidemiol 2007;17(9):669-678. [PubMed: 17512215]

4. Shah AD, Langenberg C, Rapsomaniki E, Denaxas S, Pujades-Rodriguez M, Gale CP, Deanfield J, Smeeth L, Timmis A, Hemingway H. Type 2 diabetes and incidence of cardiovascular diseases: a cohort study in 1.9 million people. The Lancet Diabetes \& Endocrinology. 2015;3(2):105-113. [PubMed: 25466521]

5. Takagi H, Takuya Umemoto for the AG. Association of diabetes mellitus with presence, expansion, and rupture of abdominal aortic aneurysm: "Curiouser and curiouser!" cried ALICE. Semin Vasc Surg 2016;29(1-2):18-26. [PubMed: 27823585]

6. Xiong J, Wu Z, Chen C, Wei Y, Guo W. Association between diabetes and prevalence and growth rate of abdominal aortic aneurysms: A meta-analysis. Int J Cardiol 2016;221:484-495. [PubMed: 27414727]

7. Lederle FA, Johnson GR, Wilson SE, Chute EP, Littooy FN, Bandyk D, Krupski WC, Barone GW, Acher CW, Ballard DJ. Prevalence and associations of abdominal aortic aneurysm detected through screening. Aneurysm Detection and Management (ADAM) Veterans Affairs Cooperative Study Group. Ann Intern Med 1997;126(6):441-449. [PubMed: 9072929]

8. Le MT, Jamrozik K, Davis TM, Norman PE. Negative association between infra-renal aortic diameter and glycaemia: the Health in Men Study. Eur J Vasc Endovasc Surg 2007;33(5):599-604. [PubMed: 17307366]

9. Blanchard JF, Armenian HK, Friesen PP. Risk factors for abdominal aortic aneurysm: results of a case-control study. American journal of epidemiology. 2000;151(6):575-583. [PubMed: 10733039]

10. Lederle FA, Larson JC, Margolis KL, Allison MA, Freiberg MS, Cochrane BB, Graettinger WF, Curb JD. Abdominal aortic aneurysm events in the women's health initiative: cohort study. BMJ (Clinical research ed) 2008;337:a1724.

11. Ohrlander T, Merlo J, Ohlsson H, Sonesson B, Acosta S. Socioeconomic position, comorbidity, and mortality in aortic aneurysms: a 13-year prospective cohort study. Ann Vasc Surg 2012;26(3):312-321. [PubMed: 22079461]

12. Takagi H, Umemoto T, Alice Group. Diabetes and Abdominal Aortic Aneurysm Growth. Angiology. 2016;67(6):513-525. [PubMed: 26311742]

13. Hjellestad ID, Softeland E, Nilsen RM, Husebye ES, Jonung T. Abdominal aortic aneurysms-glycaemic status and mortality. J Diabetes Complications. 2016;30(3):438-443. [PubMed: 26794646]

14. American Diabetes Association. Standards of Medical Care in Diabetes-2019 Abridged for Primary Care Providers. Clinical diabetes : a publication of the American Diabetes Association. 2019;37(1):11-34. [PubMed: 30705493] 
15. The Atherosclerosis Risk in Communities (ARIC) Study: design and objectives. The ARIC investigators. American journal of epidemiology. 1989;129(4):687-702. [PubMed: 2646917]

16. Selvin E, Coresh J, Zhu H, Folsom A, Steffes MW. Measurement of HbA1c from stored whole blood samples in the Atherosclerosis Risk in Communities study. Journal of Diabetes. 2010;2(2):118-124. [PubMed: 20923494]

17. Skali H, Shah A, Gupta DK, Cheng S, Claggett B, Liu J, Bello N, Aguilar D, Vardeny O, Matsushita K, Selvin E, Solomon S. Cardiac structure and function across the glycemic spectrum in elderly men and women free of prevalent heart disease: the Atherosclerosis Risk In the Community study. Circ Heart Fail. 2015;8(3):448-454. [PubMed: 25759458]

18. Levey AS, Stevens LA, Schmid CH, Zhang YL, Castro AF 3rd, Feldman HI, Kusek JW, Eggers P, Van Lente F, Greene T, Coresh J. A new equation to estimate glomerular filtration rate. Ann Intern Med 2009;150(9):604-612. [PubMed: 19414839]

19. Astor BC, Coresh J, Heiss G, Pettitt D, Sarnak MJ. Kidney function and anemia as risk factors for coronary heart disease and mortality: the Atherosclerosis Risk in Communities (ARIC) Study. American heart journal. 2006;151(2):492-500. [PubMed: 16442920]

20. Folsom AR, Yao L, Alonso A, Lutsey PL, Missov E, Lederle FA, Ballantyne CM, Tang W. Circulating Biomarkers and Abdominal Aortic Aneurysm Incidence: The Atherosclerosis Risk in Communities (ARIC) Study. Circulation. 2015;132(7):578-585. [PubMed: 26085454]

21. Tang W, Yao L, Roetker NS, Alonso A, Lutsey PL, Steenson CC, Lederle FA, Hunter DW, Bengtson LG, Guan W, Missov E, Folsom AR. Lifetime Risk and Risk Factors for Abdominal Aortic Aneurysm in a 24-Year Prospective Study: The ARIC Study (Atherosclerosis Risk in Communities). Arterioscler Thromb Vasc Biol 2016;36(12):2468-2477. [PubMed: 27834688]

22. Lopez-de-Andres A, Jimenez-Trujillo I, Jimenez-Garcia R, Hernandez-Barrera V, de Miguel-Yanes JM, Mendez-Bailon M, Perez-Farinos N, Salinero-Fort MA, Carrasco-Garrido P. National trends in incidence and outcomes of abdominal aortic aneurysm among elderly type 2 diabetic and nondiabetic patients in Spain (2003-2012). Cardiovasc Diabetol 2015;14:48. [PubMed: 25947103]

23. Larsson SC, Wallin A, Hakansson N, Stackelberg O, Back M, Wolk A. Type 1 and type 2 diabetes mellitus and incidence of seven cardiovascular diseases. Int J Cardiol 2018;262:66-70. [PubMed: 29605469]

24. Jahangir E, Lipworth L, Edwards TL, Kabagambe EK, Mumma MT, Mensah GA, Fazio S, Blot WJ, Sampson UK. Smoking, sex, risk factors and abdominal aortic aneurysms: a prospective study of 18782 persons aged above 65 years in the Southern Community Cohort Study. Journal of epidemiology and community health. 2015;69(5):481-488. [PubMed: 25563744]

25. Tornwall ME, Virtamo J, Haukka JK, Albanes D, Huttunen JK. Life-style factors and risk for abdominal aortic aneurysm in a cohort of Finnish male smokers. Epidemiology (Cambridge, Mass). 2001;12(1):94-100.

26. Wong DR, Willett WC, Rimm EB. Smoking, hypertension, alcohol consumption, and risk of abdominal aortic aneurysm in men. American journal of epidemiology. 2007;165(7):838-845. [PubMed: 17215382]

27. Campbell PT, Newton CC, Patel AV, Jacobs EJ, Gapstur SM. Diabetes and cause-specific mortality in a prospective cohort of one million U.S. adults. Diabetes care. 2012;35(9):1835-1844. [PubMed: 22699290]

28. Sode BF, Nordestgaard BG, Gronbaek M, Dahl M. Tobacco smoking and aortic aneurysm: two population-based studies. Int J Cardiol 2013;167(5):2271-2277. [PubMed: 22726392]

29. Wang L, Djousse L, Song Y, Akinkuolie AO, Matsumoto C, Manson JE, Gaziano JM, Sesso HD. Associations of Diabetes and Obesity with Risk of Abdominal Aortic Aneurysm in Men. Journal of obesity. 2017;2017:3521649. [PubMed: 28326193]

30. Tsai CL, Lin CL, Wu YY, Shieh DC, Sung FC, Kao CH. Advanced complicated diabetes mellitus is associated with a reduced risk of thoracic and abdominal aortic aneurysm rupture: a populationbased cohort study. Diabetes Metab Res Rev 2015;31(2):190-197. [PubMed: 25066630]

31. Astrand H, Ryden-Ahlgren A, Sundkvist G, Sandgren T, Lanne T. Reduced aortic wall stress in diabetes mellitus. Eur J Vasc Endovasc Surg 2007;33(5):592-598. [PubMed: 17164093] 
32. Portik-Dobos V, Anstadt MP, Hutchinson J, Bannan M, Ergul A. Evidence for a matrix metalloproteinase induction/activation system in arterial vasculature and decreased synthesis and activity in diabetes. Diabetes. 2002;51(10):3063-3068. [PubMed: 12351448]

33. Koole D, van Herwaarden JA, Schalkwijk CG, Lafeber F, Vink A, Smeets MB, Pasterkamp G, Moll FL. A potential role for glycated cross-links in abdominal aortic aneurysm disease. J Vasc Surg 2017;65(5):1493-1503 e1493. [PubMed: 27624703]

34. Kobayashi T, Osakabe T, Seyama Y. Comparison of elastolytic activity between experimental aneurysm and experimental diabetes mellitus. Biological \& pharmaceutical bulletin. 1998;21(7):775-777. [PubMed: 9703267]

35. Sakalihasan N, Heyeres A, Nusgens BV, Limet R, Lapiere CM. Modifications of the extracellular matrix of aneurysmal abdominal aortas as a function of their size. European journal of vascular surgery. 1993;7(6):633-637. [PubMed: 8270064]

36. Raffort J, Lareyre F, Clement M, Hassen-Khodja R, Chinetti G, Mallat Z. Diabetes and aortic aneurysm: current state of the art. Cardiovasc Res 2018;114(13):1702-1713. [PubMed: 30052821]

37. Fujimura N, Xiong J, Kettler EB, Xuan H, Glover KJ, Mell MW, Xu B, Dalman RL. Metformin treatment status and abdominal aortic aneurysm disease progression. J Vasc Surg 2016;64(1):4654 e48. [PubMed: 27106243]

38. Itoga NK, Rothenberg KA, Suarez P, Ho TV, Mell MW, Xu B, Curtin CM, Dalman RL. Metformin prescription status and abdominal aortic aneurysm disease progression in the U.S. veteran population. J Vasc Surg 2019;69(3):710-716 e713. [PubMed: 30197158]

39. Hsu CY, Su YW, Chen YT, Tsai SH, Chang CC, Li SY, Huang PH, Chen JW, Lin SJ. Association between use of oral-antidiabetic drugs and the risk of aortic aneurysm: a nested case-control analysis. Cardiovasc Diabetol 2016;15(1):125. [PubMed: 27585542]

40. Golledge J, Moxon J, Pinchbeck J, Anderson G, Rowbotham S, Jenkins J, Bourke M, Bourke B, Dear A, Buckenham T, Jones R, Norman PE. Association between metformin prescription and growth rates of abdominal aortic aneurysms. Br J Surg 2017;104(11):1486-1493. [PubMed: 28650557]

41. Aune D, Schlesinger S, Norat T, Riboli E. Diabetes mellitus and the risk of abdominal aortic aneurysm: A systematic review and meta-analysis of prospective studies. J Diabetes Complications. 2018;32(12):1169-1174. [PubMed: 30415876]

42. Jimenez-Quevedo P, Suzuki N, Corros C, Ferrer C, Angiolillo DJ, Alfonso F, Hernandez-Antolin R, Banuelos C, Escaned J, Fernandez C, Costa M, Macaya C, Bass T, Sabate M. Vessel shrinkage as a sign of atherosclerosis progression in type 2 diabetes: a serial intravascular ultrasound analysis. Diabetes. 2009;58(1):209-214. [PubMed: 18829988]

43. Du R, Zhang RY, Lu L, Shen Y, Pu LJ, Zhu ZB, Zhang Q, Hu J, Yang ZK, Ding FH, Zhang JS, Shen WF. Increased glycated albumin and decreased esRAGE levels in serum are related to negative coronary artery remodeling in patients with type 2 diabetes: an Intravascular ultrasound study. Cardiovasc Diabetol 2018;17(1):149. [PubMed: 30482197]

44. Laugesen E, Hoyem P, Thrysoe S, Hansen ESS, Mikkelsen AFS, Kerwin WS, Poulsen PL, Hansen TK, Kim WY. Negative Carotid Artery Remodeling in Early Type 2 Diabetes Mellitus and Increased Carotid Plaque Vulnerability in Obesity as Assessed by Magnetic Resonance Imaging. J Am Heart Assoc 2018;7(16):e008677. [PubMed: 30369319]

45. Schoenhagen P, Nissen SE, Tuzcu EM. Coronary arterial remodeling: from bench to bedside. Current atherosclerosis reports. 2003;5(2):150-154. [PubMed: 12573202]

46. Pant R, Marok R, Klein LW. Pathophysiology of coronary vascular remodeling: relationship with traditional risk factors for coronary artery disease. Cardiology in review. 2014;22(1):13-16. [PubMed: 23873211]

47. Chen XF, Wang JA, Lin XF, Tang LJ, Yu WF, Chen H, Xie XJ, Jiang JJ, Peng XH. Diabetes mellitus: is it protective against aortic root dilatation? Cardiology. 2009;112(2):138-143. [PubMed: 18600023]

48. Nardi E, Mule G, Nardi C, Geraci G, Averna M. Inverse association between type 2 diabetes and aortic root dimension in hypertensive patients. Int J Cardiol 2017;228:233-237. [PubMed: 27865191] 
49. de Vos LC, Boersema J, Hillebrands JL, Schalkwijk CG, Meerwaldt R, Breek JC, Smit AJ, Zeebregts CJ, Lefrandt JD. Diverging effects of diabetes mellitus in patients with peripheral artery disease and abdominal aortic aneurysm and the role of advanced glycation end-products: ARTERY study - protocol for a multicentre cross-sectional study. BMJ Open. 2017;7(4):e012584.

50. Patel K, Zafar MA, Ziganshin BA, Elefteriades JA. Diabetes Mellitus: Is It Protective against Aneurysm? A Narrative Review. Cardiology. 2018;141(2):107-122. [PubMed: 30453299]

51. Dattani N, Sayers RD, Bown MJ. Diabetes mellitus and abdominal aortic aneurysms: A review of the mechanisms underlying the negative relationship. Diab Vasc Dis Res 2018;15(5):367-374. [PubMed: 29874945]

52. Radak D, Tanaskovic S, Katsiki N, Isenovic ER. Protective Role of Diabetes Mellitus on Abdominal Aortic Aneurysm Pathogenesis: Myth or Reality? Current vascular pharmacology. 2016;14(2):196-200. [PubMed: 26022380]

53. Lema P, Kim J, James E St.. Overview of common errors and pitfalls to avoid in the acquisition and interpretation of ultrasound imaging of the abdominal aorta. Journal of Vascular Diagnostics and Interventions. 2017; Volume 5:41-46. 
- Diabetes, especially with longer duration, is negatively associated with the risk of abdominal aortic aneurysm (AAA);

- $\quad$ Pre-diabetes was not associated with incident AAA and showed relatively greater aortic diameter compared to non-diabetes.

- $\quad$ Reduced aortic diameter is likely to be a structural mechanism of decreased AAA risk in diabetes. 


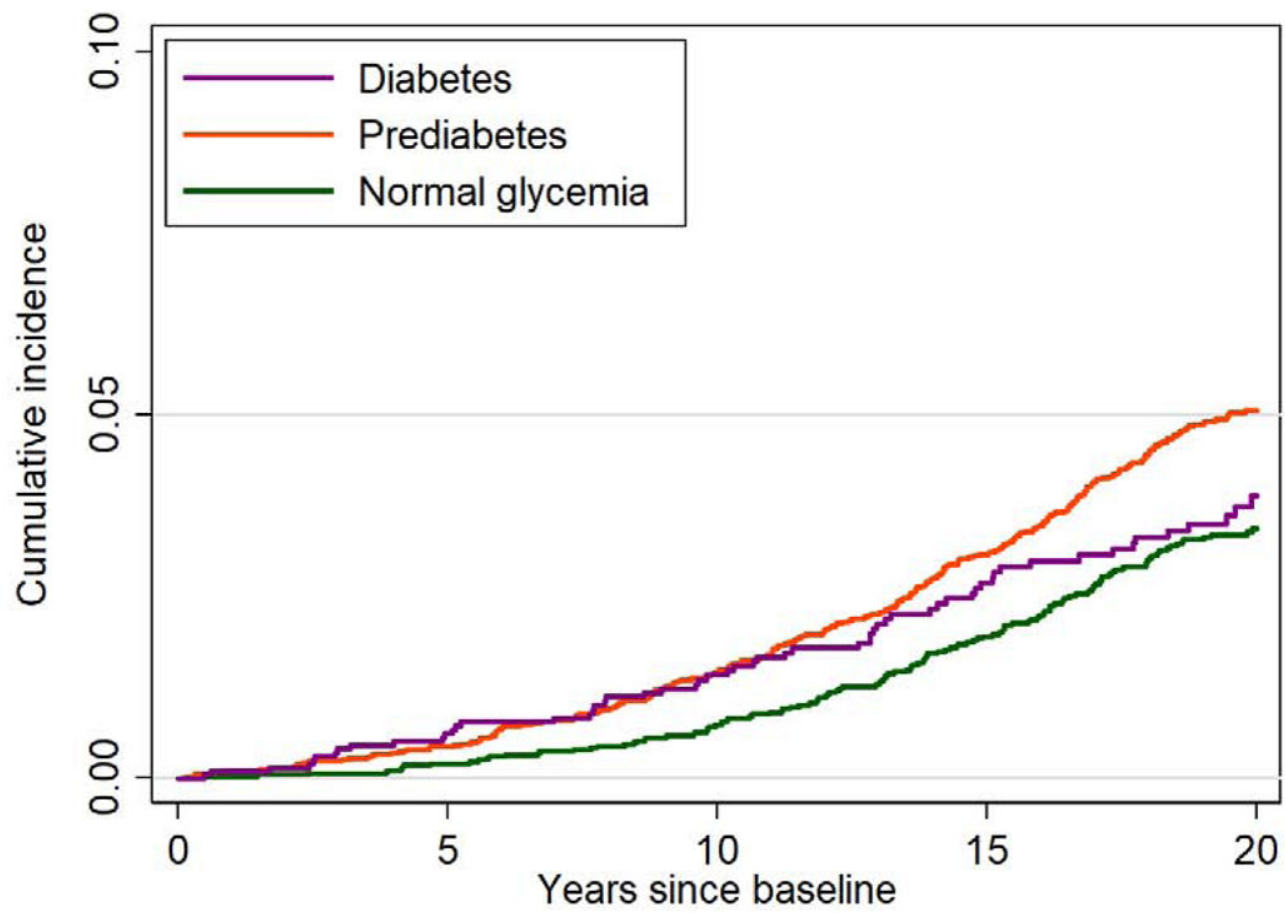

\section{Number at risk}

Diabetes 2036

Prediabetes 6684

Normal glycemia 4396

1851

1628

1325

616

6385

5922

5292

4267

4061

3737

2956

2067

Figure 1.

Cumulative abdominal aortic aneurysm (AAA) incidence by glycemic status at visit 2 


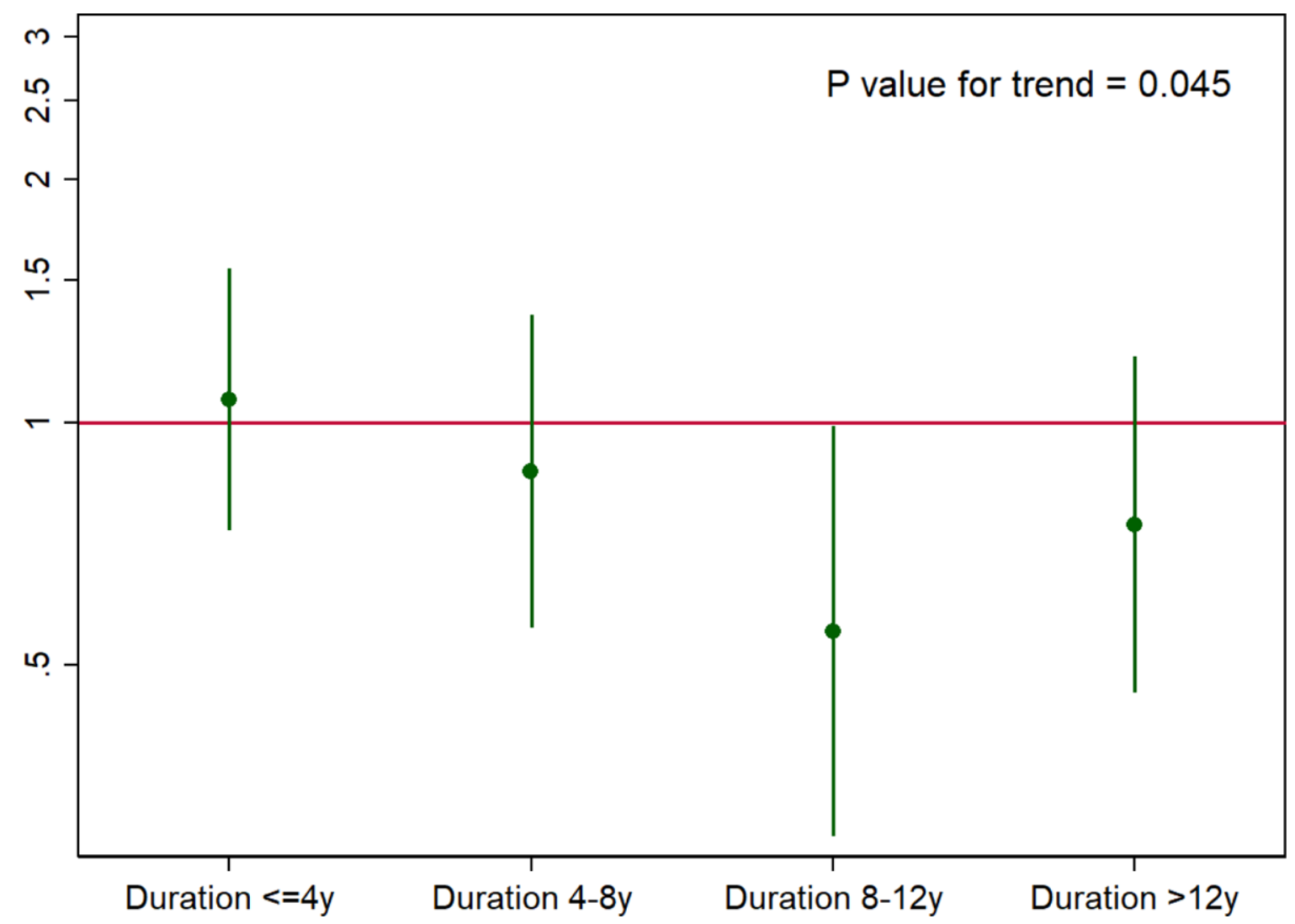

Figure 2.

Adjusted hazard ratio of incident abdominal aortic aneurysm (AAA) according to the duration of diabetes among incident cases as a time-varying exposure.

Hazard ratios were adjusted for updated covariates in Model 2: age, sex, race, education, BMI, height, total cholesterol, HDL cholesterol, systolic blood pressure, diastolic blood pressure, anti-hypertensive medication, cholesterol-lowering medication, cigarette packyears, alcohol drinking, eGFR, prevalent peripheral artery disease, prevalent coronary heart disease, and prevalent stroke. 


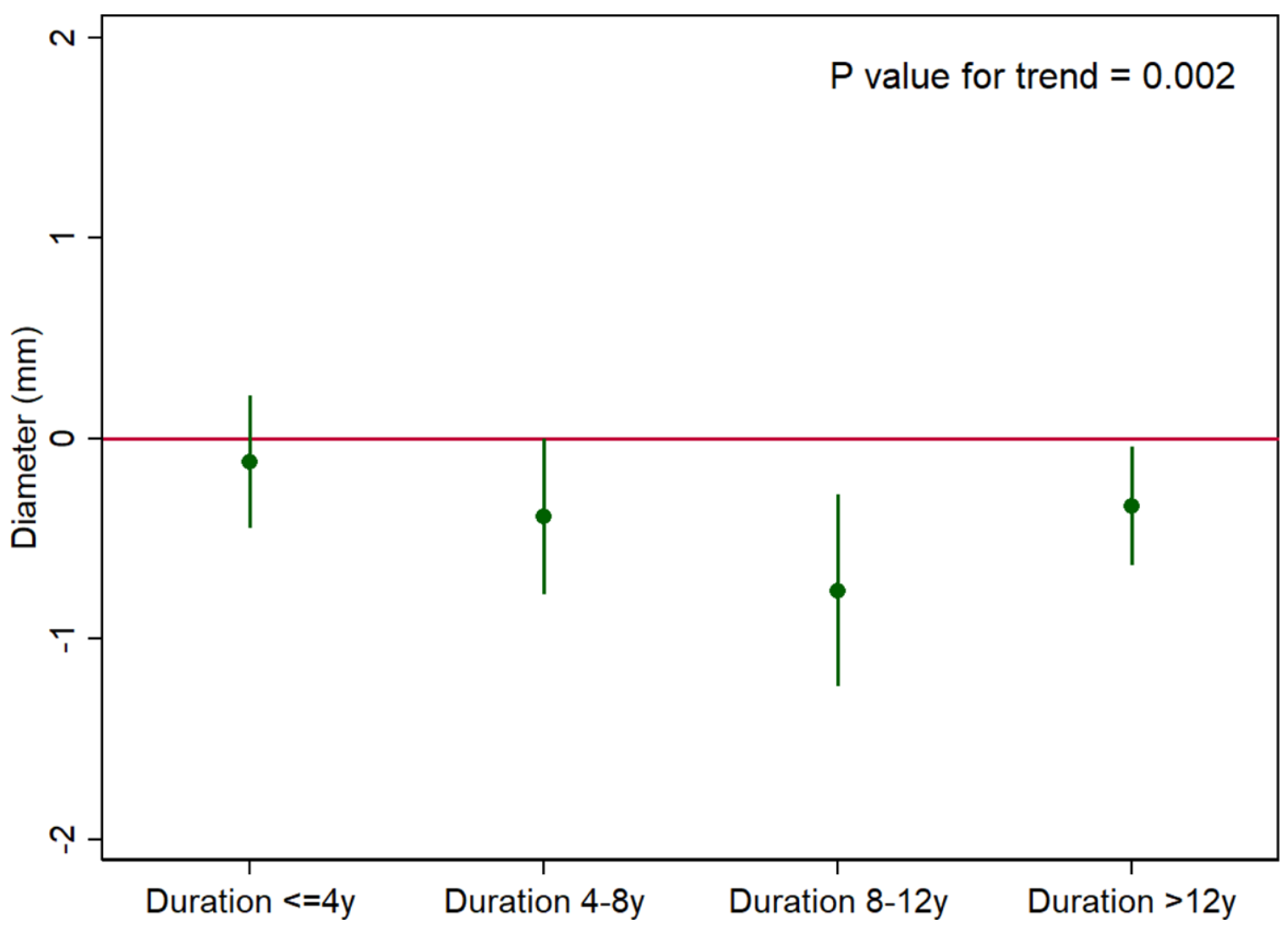

Figure 3.

Adjusted difference in maximum diameter $(\mathrm{mm})$ for diabetes vs. non-diabetes by cumulative duration of diabetes.

Hazard ratios were adjusted for covariates in Model 2: age, sex, race, education, BMI, height, total cholesterol, HDL cholesterol, systolic blood pressure, diastolic blood pressure, anti-hypertensive medication, cholesterol-lowering medication, cigarette pack-years, alcohol drinking, eGFR, prevalent peripheral artery disease, prevalent coronary heart disease, and prevalent stroke. 
Table 1.

Baseline characteristics at visit 2 according to glycemic status, ARIC, 1990-1992.

\begin{tabular}{|c|c|c|c|c|}
\hline & Normal glycemia & Prediabetes & Diabetes & $p$ value \\
\hline $\mathrm{N}=13,116$ & 4,396 & 6,684 & 2,036 & \\
\hline Age & $56.2(5.6)$ & $57.2(5.7)$ & $58.1(5.7)$ & $<0.001$ \\
\hline Female & $2,886(65.7)$ & $3,306(49.5)$ & $1,110(54.5)$ & $<0.001$ \\
\hline White & $3,724(84.7)$ & $5,099(76.3)$ & $1,225(60.2)$ & $<0.001$ \\
\hline BMI, $\mathrm{kg} / \mathrm{m}^{2}$ & $26.0(4.5)$ & $28.2(5.2)$ & $31.1(6.0)$ & $<0.001$ \\
\hline Height, $\mathrm{cm}$ & $167.0(8.9)$ & $169.4(9.5)$ & $168.3(9.1)$ & $<0.001$ \\
\hline Education level & & & & $<0.001$ \\
\hline Basic Education & $653(14.9)$ & $1,442(21.6)$ & $671(33.0)$ & \\
\hline Intermediate Education & $1,934(44.0)$ & $2,750(41.1)$ & $796(39.1)$ & \\
\hline Advanced Education & $1,809(41.2)$ & $2,492(37.3)$ & $569(27.9)$ & \\
\hline Cigarette pack-years & $12.9(19.5)$ & $16.3(21.2)$ & $17.0(23.9)$ & $<0.001$ \\
\hline Alcohol drinking & & & & $<0.001$ \\
\hline Current & $2,690(61.2)$ & $3,935(58.9)$ & $850(41.7)$ & \\
\hline Former & $743(16.9)$ & $1,338(20.0)$ & $625(30.7)$ & \\
\hline Never & $963(21.9)$ & $1,411(21.1)$ & $561(27.6)$ & \\
\hline Systolic blood pressure, $\mathrm{mmHg}$ & $117.3(18.1)$ & $122.1(18.1)$ & $127.9(19.3)$ & $<0.001$ \\
\hline Diastolic blood pressure, $\mathrm{mmHg}$ & $70.8(10.2)$ & $72.9(10.1)$ & $72.8(10.5)$ & $<0.001$ \\
\hline HDL cholesterol, mg/dL & $54.4(17.3)$ & $48.4(16.1)$ & $43.3(14.1)$ & $<0.001$ \\
\hline Total cholesterol, $\mathrm{mg} / \mathrm{dL}$ & $207.0(37.5)$ & $210.3(38.8)$ & $215.0(44.7)$ & $<0.001$ \\
\hline eGFR, $\mathrm{mL} / \mathrm{min} / 1.73 \mathrm{~m}^{2}$ & $96.8(14.0)$ & $95.7(15.3)$ & $96.9(19.4)$ & $<0.001$ \\
\hline Anti-hypertensive medication & $955(21.7)$ & $2,190(32.8)$ & $1,108(54.4)$ & $<0.001$ \\
\hline Cholesterol lowering medication & $223(5.1)$ & $431(6.4)$ & $197(9.7)$ & $<0.001$ \\
\hline Prevalent coronary heart disease & $176(4.0)$ & $365(5.5)$ & $211(10.4)$ & $<0.001$ \\
\hline Prevalent stroke & $56(1.3)$ & $104(1.6)$ & $80(3.9)$ & $<0.001$ \\
\hline Prevalent peripheral artery disease & $239(5.4)$ & $514(7.7)$ & $306(15.0)$ & $<0.001$ \\
\hline
\end{tabular}

Statistics are presented as mean (SD) or $\mathrm{n}(\%)$.

Abbreviations: BMI, body mass index; HDL, high density lipoprotein; eGFR, estimated glomerular filtration rate.

SI conversion factors: To convert total and HDL cholesterol to mmol/L, multiple by 0.0259 . 
Table 2.

Adjusted hazard ratio of incident AAA by glycemic status and follow up time, ARIC, HR (95\%CI)

\begin{tabular}{|c|c|c|}
\hline Glycemic status & Model 1 & Model 2 \\
\hline & \multicolumn{2}{|c|}{ All follow up time, $N=13,116$} \\
\hline Normal glycemia & 1 (ref) & $1(\mathrm{ref})$ \\
\hline Prediabetes & $1.54(1.25,1.89)$ & $1.06(0.85,1.30)$ \\
\hline \multirow[t]{2}{*}{ Diabetes } & $1.20(0.89,1.63)$ & $0.71(0.51,0.99)$ \\
\hline & \multicolumn{2}{|c|}{ Follow up time $\leq 10$ years, $N=13,116$} \\
\hline Normal glycemia & 1 (ref) & 1 (ref) \\
\hline Prediabetes & $1.99(1.33,3.00)$ & $1.28(0.84,1.95)$ \\
\hline \multirow[t]{2}{*}{ Diabetes } & $1.96(1.17,3.31)$ & $1.06(0.61,1.85)$ \\
\hline & \multicolumn{2}{|c|}{ Follow up time $>10$ years, $N=11,611$} \\
\hline Normal glycemia & 1 (ref) & 1 (ref) \\
\hline Prediabetes & $1.40(1.10,1.77)$ & $0.98(0.77,1.25)$ \\
\hline Diabetes & $0.94(0.64,1.39)$ & $0.58(0.38,0.87)$ \\
\hline
\end{tabular}

Model 1 unadjusted

Model 2 adjusted for age, sex, race, education, BMI, height, total cholesterol, HDL cholesterol, systolic blood pressure, diastolic blood pressure, anti-hypertensive medication, cholesterol-lowering medication, cigarette pack-years, alcohol drinking, eGFR, prevalent peripheral artery disease, prevalent coronary heart disease, and prevalent stroke 